\title{
Testing post-weaning food motivation in low and normal birth weight pigs in a runway and operant conditioning task
}

\author{
L.M. van Eck ${ }^{\mathrm{a}, *}$, A. Antonides ${ }^{\mathrm{b}, \mathrm{c}}$, R.E. Nordquist ${ }^{\mathrm{b}, \mathrm{c}}$, F.J. van der Staay ${ }^{\mathrm{b}, \mathrm{c}}$ \\ a Adaptation Physiology Group, Wageningen University, The Netherlands \\ ${ }^{\mathrm{b}}$ Behaviour and Welfare Group (Formerly: Emotion and Cognition Group), Department of Farm Animal Health, Faculty of Veterinary Medicine, Utrecht \\ University, P.O. Box 80151, 3508 TD Utrecht, The Netherlands \\ ${ }^{\mathrm{c}}$ Brain Center Rudolf Magnus, Utrecht University, Universiteitsweg 100, 3584 CG Utrecht, The Netherlands
}

\section{A R T I C L E I N F O}

\section{Article history:}

Received 23 January 2016

Received in revised form 11 May 2016

Accepted 15 May 2016

Available online 19 May 2016

\section{Keywords:}

Motivation

Pigs

Birth weight

Runway

Operant conditioning

\begin{abstract}
A B S T R A C T
Low birth weight (LBW) pigs face more welfare challenges than their normal birth weight (NBW) siblings. Understanding the underlying mechanisms of cognitive and learning abilities in these pigs may help to improve their welfare. Early competition in life over resources, combined with the higher need for nutrient intake, make it likely that LBW pigs have a higher motivation for food than NBW pigs. This study aimed to compare the motivation to obtain food rewards between LBW and NBW pigs, using variable numbers of rewards in two separate tasks; a runway and an operant conditioning task (the nose wheel task). Ten pairs of littermates were used. From each litter, one low birth weight piglet (mean birth weight \pm SEM: $854 \pm 33 \mathrm{~g}$ ) and one normal birth weight piglet (1332 $\pm 53 \mathrm{~g}$ ) was selected. Pigs were tested in the runway task at 12 weeks of age and the operant nose wheel task at 19 weeks of age. Both tasks consisted of a baseline phase (two rewards), a high reward phase (eight rewards) and an extinction phase (no rewards). Statistical analyses using mixed models showed that NBW animals left the start box faster than LBW animals in the high reward phase in the runway task. However, their run time in this phase was not shorter and no other birth weight effects were found in any other phase or measure in this task nor in the nose wheel task. All animals decreased their run time in the runway task between the baseline phase and high reward phase, and increased their run time in the extinction phase $(p<0.05)$. Likewise, in the nose wheel task, all animals reached a higher number of total rewards gained and spent a lower percentage of time away from the feeder in the high reward phase compared to the baseline phase $(\mathrm{p}<0.05)$. Additionally, they showed a decrease in motivation during the extinction phase $(\mathrm{p}<0.05)$. Our results indicate that there is no difference in motivation to obtain food rewards between LBW and NBW pigs. However, both the results of the runway and the nose wheel task show a phase effect between the baseline, high reward and the extinction phase. This is in accordance with the underlying theory that animals have a higher motivation for resources that are more desired. Therefore, we show that both tasks are sensitive enough to measure motivation for food rewards in pigs, and are consequently useful to study factors influencing pig motivation.
\end{abstract}

(c) 2016 Elsevier B.V. All rights reserved.

\section{Introduction}

In current pig husbandry, there is a strong selection for high sow fecundity. This has resulted in an average litter size increase of 0.25 piglets per year (Hazeleger et al., 2007). Consequently, there is an

\footnotetext{
* Corresponding author at: Behaviour and Welfare Group, Department of Farm Animal Health, Faculty of Veterinary Medicine, Utrecht University, P.O. Box 80151, 3508 TD, The Netherlands.

E-mail address: lieskevaneck@gmail.com (L.M. van Eck).
}

increased birth weight variation within litters (Rutherford et al., 2013). The mean birth weight of a litter decreases with approximately $35 \mathrm{~g}$ for each piglet additionally born in a litter (Quiniou et al., 2002). This causes an increased incidence of piglets that are born with a low birth weight (LBW).

LBW in piglets has several negative consequences for their survival and welfare. Pigs experience both pre- and postnatal competition for resources. Colostrum intake as soon as possible after birth is essential for piglet survival, which is difficult for LBW piglets as they need to compete with their larger siblings for teats. Teat competition is accompanied by aggression, and usually the piglets 
with higher birth weights win these fights (Scheel et al., 1977). LBW piglets are slower to respond to their environment than normal birth weight (NBW) piglets, causing increased early mortality risks due to crushing by the sow (Baxter et al., 2008). Additionally, LBW piglets have lower body reserves and a higher risk to suffer from hypothermia and starvation (Herpin et al., 2002). Moreover, weight gain is reduced lifelong in LBW piglets (Douglas et al., 2013).

Understanding the underlying mechanisms of cognitive abilities in pigs may help to improve their welfare (Gieling et al., 2011). Although many studies have investigated the effects of LBW on production and welfare (e.g. Baxter et al., 2008), to our knowledge, no studies have yet examined the effects of LBW in pigs on food motivation.

There are some indications that LBW pigs have an increased motivation for food. Murphy et al. (2013) found that LBW piglets learned a discrimination task faster than NBW pigs, hypothesized to be due to higher (food) motivation. The results of another study on LBW pigs' cognitive functioning showed that LBW piglets outperformed their NBW siblings in a spatial cognitive holeboard task (Antonides et al., 2015). The authors argued that this may have been due to increased motivation for food.

In studies on both rats and humans it has been found that LBW alters feeding preferences. LBW rats have a higher overall food intake (Vickers et al., 2000). LBW (in humans: small for gestational age, SGA) individuals who experienced prenatal nutrient restriction showed a higher preference for fatty diets (Lussana et al., 2008) and SGA women favor carbohydrates over proteins in their diet (Barbieri et al., 2009). It has been suggested that restricted nutrient supply and a stressful prenatal environment prepare the body for the postnatal environment by adjusting metabolic patterns that favor energy storage (Gluckman and Hanson, 2004). In livestock, prenatal stress caused by malnutrition has an impact on health and behavior in later life (Rutherford et al., 2012). Hair cortisol as a longterm measure for stress can provide an indication of the differences in the stress physiology between LBW and NBW piglets.

The early competition over resources, combined with the possible higher need for energy intake, make it likely that LBW piglets have a higher motivation for food (rewards) than NBW pigs. The current study aimed to compare the motivation to obtain food rewards between LBW and NBW pigs. To this end, we subjected ten LBW pigs from different litters and their ten NBW siblings to two food motivation tasks. Different tools can be used to induce a cost for a desired resource in order to measure motivation (Kirkden and Pajor, 2006). In the first task, food motivation was assessed in a runway. In the second task, the pigs' motivation was tested using a nose wheel in a feeding station as a cost mechanism to gain access to the reward (da Silva et al., 2012).

We expected to find a higher food motivation in LBW pigs than in NBW pigs in both tasks. As animals show a higher motivation for more desired resources (Kirkden and Pajor, 2006), we expected all pigs to show increased motivation for a higher number of rewards, and a decrease in motivation when no rewards were offered (extinction). Additionally, we measured hair cortisol as a long-term measure for stress. In accordance with previous findings in LBW pigs' hair cortisol (Antonides et al., 2015), we expected the LBW pigs to have lower hair cortisol values than NBW pigs.

\section{Materials and methods}

\subsection{Ethical note}

This study was reviewed and approved by the local ethics committee (DEC, DierExperimenten Commissie) and is in accordance with the recommendations of the EU directive 86/609/EEC.

\subsection{Animals and housing}

Ten NBW and ten LBW pigs [Duroc $\times$ (Terra $\times$ Finnish landrace)], born in conventional farrowing crates on the commercial pig breeding farm of Utrecht University were selected. Ten litters in which at least 10 piglets were born were used, born in two batches of five litters each in two successive weeks, to ensure that enough LBW piglets could be selected. All piglets of each litter were weighed on the day of birth, including all stillborn piglets and piglets that died shortly after birth. From each litter, one LBW piglet was selected, with a maximum weight of 1 SD below the average birth weight of 484 piglets weighed in previous experiments. This yielded a maximum weight of $1050 \mathrm{~g}$ for the LBW piglets. Moreover, the selected piglets were at least 1 SD below the average birth weight of the litter they were born into. The lightest piglet of the litter which met both criteria and that was healthy and lively was selected from each litter. The selected NBW sibling was the piglet with a birth weight closest to the average birth weight of the litter. Preferably, NBW and LBW pigs with the same sex were selected per litter. For one pair, no NBW sibling with the same sex as the selected LBW pig close to the birth weight average was born. Therefore, the final selection consisted of five pairs of males, four pairs of females and one male-female pair. For two pairs, the originally selected NBW piglet was crushed by the sow. Selection of the new NBW piglet was based on the weight closest to the average of the litter on week four instead of day one, since birth weight information of the remaining NBW pigs in the litter was not saved at the time. As the weight of LBW piglets remains lower compared to NBW piglets throughout life (Douglas et al., 2013; Antonides et al., 2015), we assumed that these two pigs did not have a LBW. To check for asymmetrical growth as an indicator of intra-uterine growth retardation (IUGR), head size (snout to back of cranium) and total body length (snout to tail base) were measured on the day of birth (Amdi et al., 2013).

Selected piglets were weaned at four weeks of age and housed in groups of five of the same age, with NBW and LBW piglets housed separately. This was done to avoid strong competition for hierarchy within the pen. Since the NBW and LBW pigs were thus separated, littermates were divided over groups and not housed together. Groups were housed in four similar pens (ca. $5 \mathrm{~m} \times 6 \mathrm{~m}$ ) with concrete flooring, with straw bedding and toys. Each pen contained a covered piglet nest containing a rubber mat and a thick layer of sawdust and straw bedding. A heat lamp was suspended approximately $1 \mathrm{~m}$ above ground in the piglet nest to avoid chilling of the piglets. The heat lamps were removed at 14 weeks of age, by which time the pigs were no longer observed lying under the lamps or showing huddling behavior. Lights in the stable were on between 7:30 h and 16:30 h. Ambient temperature in the stable was recorded daily during the experiment and ranged from $-1{ }^{\circ} \mathrm{C}$ to $14^{\circ} \mathrm{C}$. The stable was naturally ventilated (no heating aside from the above-mentioned heat lamps). Water and feed was provided ad libitum, except for two hours prior to testing when pigs were mildly food deprived by removing all feed. This was done to prevent saturation before testing. Feed was offered in a large feeding trough $(2.5 \mathrm{~m} \times 0.3 \mathrm{~m})$ to avoid feeding aggression. Radios played continuously at the pens and in the testing area at a moderate volume to prevent sudden background noises from startling the pigs during testing. Pigs were weighed on the day of birth and then weekly from week four until week 20. Each task was performed in a separate test area with an adjacent waiting area.

After weaning and prior to testing, all pigs were habituated to the experimenters, waiting areas and testing areas for approximately one hour per day. The waiting and testing areas were located in the same building as the home pens. At the age of 12 weeks, all pigs were tested in the runway task. At the age of 19 weeks, pigs were tested in the nose wheel task (progressive ratio task). 
At the age of 22 weeks, all pigs were transported to a commercial slaughterhouse.

\subsection{The runway task}

In the runway task, pigs needed to walk or run from a start box to a goal box to obtain food rewards (M\&M's ${ }^{\circledR}$ chocolates). The runway $(6 \mathrm{~m} \times 1 \mathrm{~m})$ had two boxes $(1 \mathrm{~m} \times 1 \mathrm{~m})$ on each side that served either as start box or goal box in each run (Fig. 1). The boxes had an upward sliding door (width: $1 \mathrm{~m}$ ) allowing access to the runway. Two experimenters operated the doors from outside the runway, using a rope and pulley system. Which experimenter operated which box was alternated daily. Both boxes contained a reward box $(40 \mathrm{~cm} \times 40 \mathrm{~cm})$ with a concrete food bowl in it. The grey synthetic walls of the runway and boxes were $80 \mathrm{~cm}$ high.

Pigs were gradually habituated and trained in the runway. This was initially done in pairs to minimize stress and, once the pigs did not show escape attempts or freezing behavior anymore, pigs were trained individually. During these training sessions, pigs entered the runway and were offered two rewards in each food bowl, which they could approach at will. Once the pigs had learned where to find the reward, the upward sliding doors were closed as soon as the pigs had entered the box, and opened again when the pigs had consumed the rewards. At 12 weeks of age, when all pigs successfully completed runs and showed no fear of the sliding doors anymore, formal testing started.

Before a session started, a pig was led into the runway and entered the first start box (first start box was alternated daily between box 1 and 2). To motivate pigs to enter the start box, they were rewarded with the same amount of rewards they would receive in each run that day (two or eight, see below). The door of the other box was kept closed to direct the pig to the correct start box. As soon as the pig entered the start box, the sliding door was lowered. After the rewards were consumed, both sliding doors were opened and a session started. Each session consisted of four consecutive runs. A run consisted of a piglet leaving the start box and entering the goal box where the rewards were consumed. When a pig reached the food bowl of the goal box, both sliding doors were closed. The experimenter operating the start box of that session refilled the food bowl with rewards for the next run. As soon as all rewards in the goal box were consumed, both doors were opened again for the next run. After completing four runs (one session), the door of the last goal box was opened and the piglet was allowed to leave the runway through a door on the side (see Fig. 1). Pen mates were housed in the adjacent waiting area during testing. Each day, pigs of a pen were tested in the same order (pen and pig order were randomized daily). All pigs received three sessions (i.e. 12 runs) per day.

\subsubsection{Phases in the runway task}

The runway task was performed in five different phases, over seven (working) days. In the first phase (baseline, three days, nine sessions in total), two rewards were used in each run. In the second phase (high reward, two days, six sessions in total), the number of rewards was increased to eight, while all other test procedures remained the same. For the third phase (obstacles, one day, three sessions), obstacles were added to the runway to increase the difficulty to complete a run. In this phase, eight rewards were given in each run. The four obstacles were: a yellow garbage bin weighed down with bricks, two small feeding towers placed upside down, and a chair placed upside down. Only the chair completely blocked the runway, thus pigs had to climb over it. In the fourth phase (rebaseline, one day, three sessions), the obstacles were removed and two rewards were given per run. The re-baseline phase was added to 'reset' the motivation of the pigs before the extinction phase, as their motivation could have otherwise been influenced by the obstacles being removed from the runway in the extinction phase.

In the fifth and last phase (extinction, one day, two sessions), no rewards were offered. The extinction phase consisted of two instead of three sessions, as pigs failed to complete the runs, indicating rapid extinction of their motivation.

\subsubsection{Recorded data in the runway task}

All sessions were recorded with two camcorders (JVC Adixxion, GC-XA1) that were mounted $1 \mathrm{~m}$ above each goal box. Behaviors were scored from the recordings frame-by-frame with accuracy to a tenth of a second, using Microsoft Windows Movie Maker. Each of the observers scored half of the videos. In addition, in order to calculate the inter-rater reliability using Spearman's rank correlations test (Martin and Bateson, 1993) an overlap of $15 \%$ of all videos (of different phases) was scored independently by both observers. Inter-rater reliability was very high $(r>0.99 ; \mathrm{p}<0.01)$.

Recorded measures were: (1) leave time: the time in seconds elapsed between retraction of the pig's head from the food bowl and leaving the start box with all four legs; (2) run time: the time in seconds between leaving the start box with all four legs and the snout reaching the food bowl in the goal box; and (3) session duration: the time in seconds elapsed between leaving the start box with four legs in the first run and the snout reaching the food bowl in the goal box in the last run. When a run lasted more than $60 \mathrm{~s}$, pigs were encouraged by the experimenters to reach the goal box and the maximum run time of $60 \mathrm{~s}$ was scored. When the maximum time was scored for more than two consecutive runs, a session was ended. This was done in two sessions of one NBW pig, for which missing values were assigned in the remaining runs.

\subsection{The nose wheel task}

At the age of 19 weeks, all pigs were tested for their motivation to work for a food reward in the nose wheel task. To this end, a nose wheel feeding station was used (developed by Verbakel B.V., The Netherlands), as described by da Silva et al. (2012). The nose wheel feeding station was a custom-made feeder with a rotation disc. In order to receive a reward, a pig needed to push the rotating disc $360^{\circ}$ in an upward direction with their snout. M\&M's ${ }^{\circledR}$ chocolates were used as reward, and were delivered manually into the feeder by the experimenter standing next to the feeding station, after the required number of rotations was completed. An automated loud click-sound indicated that the required number of rotations was completed and that the pig would receive a reward. The feeding station was located in a test room measuring $3 \mathrm{~m} \times 2 \mathrm{~m}$, where the pig could move freely during the experiment. Testing was done using a progressive ratio schedule. This means that, each time a reward was received, the number of required wheel turns was automatically increased with one rotation (by a connected computer) in order to gain the next reward, starting at one rotation for the first reward.

All pigs were trained to operate the nose wheel feeding station in a fixed ratio schedule and then in a progressive ratio (PR) schedule. Testing started when all pigs could operate the nose wheel successfully to gain rewards. Success was determined by rewards being earned by the pigs six consecutive times (with the last reward requiring six full turns of the wheel).

\subsubsection{Phases in the nose wheel task}

The nose wheel task was conducted in three different phases divided over five testing days. In the first phase (baseline, two days) two M\&M's ${ }^{\circledR}$ chocolates were used as reward. In the second phase (high reward, two days) eight M\&M's ${ }^{\circledR}$ were used as reward. In the last phase (extinction, one day) all test procedures remained the same, but no reward was given. During the extinction phase, 


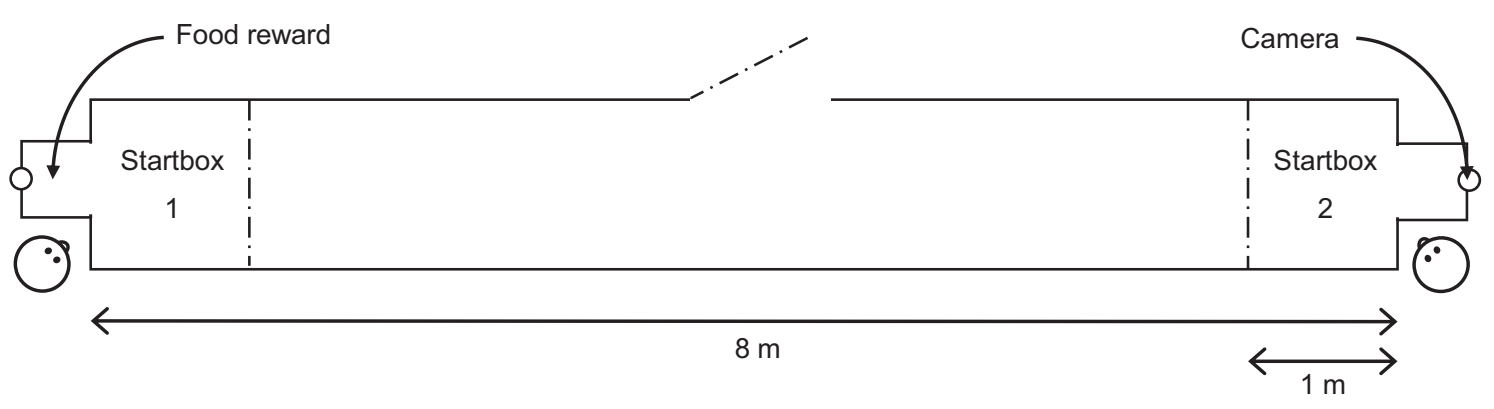

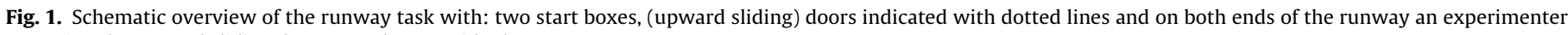
operating the upward sliding doors, standing outside the runway.

the loud click-sound still indicated that the required number of rotations was performed.

\subsubsection{Recorded data in the nose wheel task}

Durations in the nose wheel task were recorded live during trials by two experimenters using stopwatches. A trial started when a pig first touched the rotation disk with its snout. The following measures were recorded or calculated: (1) time away from feeder (in seconds), i.e. the time during a trial that the pig was not operating the feeding station (head turned away from the feeding station); (2) number of times rewarded, i.e. the number of times the pig was rewarded for completing the required number of wheel turns during a trial; (3) trial time (in seconds), i.e. the time that elapsed between when the rotation disc was first touched and when a pig was away from the feeder for longer than 120 s, or after the maximum time of $900 \mathrm{~s}$ had elapsed; (4) average time (in seconds) per turn, i.e. the average time for one complete rotation of the nose wheel disc, calculated as (trial time - time away from feeder)/number of completed rotations. For example, when the number of times rewarded $=6$, then the number of completed rotations was $(1+2+3+4+5+6)=21$; and (5) percentage of time away from feeder, calculated as (time away from feeder/trial time) $\times 100$.

\subsection{Hair samples for measuring cortisol}

At 12 weeks of age, hair samples $(0.5-1 \mathrm{~g})$ were taken from the left flank of each animal with a trimmer. To this end, animals were briefly restrained with a pig snare. Of each hair sample, $250 \mathrm{mg}$ was washed, dried and ground with a bead beater for $30 \mathrm{~min}$ in $2 \mathrm{ml}$ Eppendorf tubes containing three $1 \mathrm{~mm}$ steal beads. Thereafter, $50 \mathrm{mg}$ of each powdered sample was collected in a micro-centrifuge tube. One $\mathrm{ml}$ methanol was added after which the samples were incubated at room temperature for $24 \mathrm{~h}$ with slow rotation to extract steroids. Of the extract, $0.6 \mathrm{ml}$ was placed in a new tube and dried at $45^{\circ} \mathrm{C}$ in a heating block overnight. The dried extracts were dissolved in $0.4 \mathrm{ml}$ phosphate buffer. Cortisol concentrations were then determined in duplicate, using a Salivary Cortisol ELISA kit (Salimetrics LLC, PA, USA). Because not enough hair for analysis could be collected from one NBW animal, analyses were performed on data of ten LBW and nine NBW pigs.

\subsection{Statistical analyses}

All data were analyzed using the statistical software program SAS (version 9.4, SAS Institute, Cary, NC, USA). First, residuals of all variables were tested for normality using the Shapiro-Wilk test (SAS PROC UNIVARIATE). Variables expressing latencies or durations were $\log _{10}$-transformed to meet the normality assumption. In all mixed model analyses, a random effect for litter was added to account for clustering of piglets within litters.
The effects of birth weight on the growth curves were analyzed with a mixed model ANOVA to account for repeated measures, with the fixed effects Birth weight (LBW or NBW), Week, and their interaction (SAS PROC MIXED). The effects of birth weight on head length in $\mathrm{cm}$, full body length in $\mathrm{cm}$, head length as percentage of full body length and cortisol concentration in hair samples at 12 weeks of age were analyzed using a mixed model ANOVA with the fixed effect Birth weight.

\subsubsection{The runway task analyses}

The mean values for leave time and run time were calculated for each pig over all runs of one day. Session duration was averaged over all sessions per day. The runway data analyses were performed for the five different phases: baseline, high reward, obstacles, rebaseline and extinction. Effects of birth weight on all variables were analyzed using a mixed model ANOVA per day (starting on the third baseline day). Additionally, changes over the three baseline days were analyzed. Differences between the third baseline day and the high reward phase, the baseline and re-baseline phase and between the re-baseline and extinction phase were analyzed. Fixed effects for these analyses were Birth weight, Day and their interaction.

For the obstacle and extinction phase, effects on leave time and run time were analyzed over the different sessions. For the obstacle phase, this was done to see if the pigs decreased their average run time over sessions. For the extinction phase, this was done to see if animals became less motivated in the second session. For these analyses, fixed effects were Birth weight, Session and their interaction.

\subsubsection{The nose wheel task analyses}

Data from the nose wheel task were analyzed using a mixed model ANOVA with fixed effects Birth weight, Day and their interaction. To assess possible learning effects over the days, effects during the baseline phase (two days) and the high reward phase (two days) were analyzed. Furthermore, effects of the transition between the phases were analyzed. To this end, the second baseline day was compared to the first high reward day, and the second high reward day to the extinction phase (one day).

The total trial duration was not normally distributed, as data were cut off with a maximum trial duration of $900 \mathrm{~s}$, which was reached in $85 \%$ of all trials. All trials were therefore categorized as trial duration $<900 \mathrm{~s}$ (score 0 ), or as maximum trial duration reached $=900 \mathrm{~s}$ (score 1 ). The data were then analyzed using the Fisher exact probability test.

\section{Results}

\subsection{Birth weights and growth}

NBW piglets had higher birth weights (Fig. $2 \mathrm{~A} ; \mathrm{F}_{1,7}=120.58$; $\mathrm{p}<0.001)$, larger heads $\left(\mathrm{F}_{1,7}=37.49 ; \mathrm{p}<0.001 ;\right.$ mean \pm SEM: 

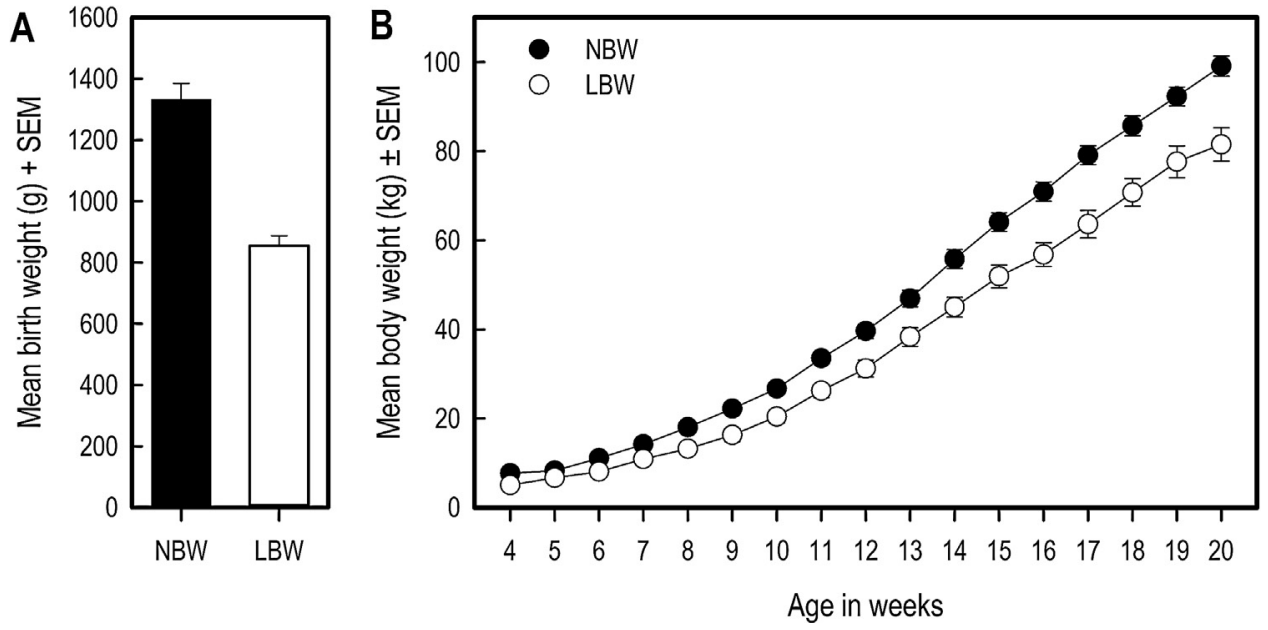

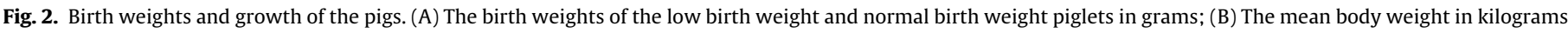
of all pigs from weaning at four weeks until 20 weeks of age.

NBW $11.00 \pm 0.19 \mathrm{~cm}$; LBW $9.83 \pm 0.12 \mathrm{~cm}$ ) and larger bodies at birth $\left(F_{1,7}=23.35 ; p=0.002 ; N B W 34.31 \pm 0.63 \mathrm{~cm}\right.$; LBW $31.17 \pm 0.45 \mathrm{~cm})$ than LBW piglets. The head size relative to the full body length did not differ between the groups $\left(F_{1,7}=0.32 ; p=0.590\right.$; NBW $32.12 \pm 0.74 \%$; LBW $31.60 \pm 0.61 \%$ ). The weights of LBW pigs remained, on average, lower than those of NBW pigs during the experiment (birth weight: $F_{1,303}=262.61$; $p<0.001$; Fig. 2B). Furthermore, NBW pigs gained weight faster than LBW pigs (birth weight by week interaction: $F_{17,303}=6.06 ; p<0.001$ ).

\subsection{The runway task}

In the high reward phase, NBW pigs had a shorter leave time (birth weight: $F_{1,9}=9.10 ; p=0.010$ ) than LBW pigs (Fig. 3A). Leave time tended to decrease in NBW pigs, but not in LBW pigs, in the transition from the baseline to the high reward phase (birth weight by day interaction: $F_{1,27}=4.12 ; p=0.050$ ). This is in line with the difference between birth weight groups in leave time found in the high reward phase. No other birth weight effects within or between phases were found in this task.

\subsubsection{Phase effects}

During the baseline phase (three days), both run time (day: $F_{2,45}=4.98 ; p=0.010$ ) and session duration (day: $F_{2,45}=3.91$; $\mathrm{p}=0.030$ ) decreased for all animals. There was no change in leave time during the three baseline days. No difference was found between the baseline and re-baseline phase for leave time, run time and session duration for all animals. In the high reward phase, overall the animals decreased their run time (day: $F_{1,27}=6.25 ; p=0.020$; Fig. 3B) and session duration (day: $F_{1,27}=13.62 ; p=0.001$ ) compared to the last baseline day. The day effects found within the baseline phase were smaller than the phase effects. Between the rebaseline and extinction phase, all animals increased their leave time (day: $F_{1,27}=15.53 ; p<0.001$; Fig. $3 A$ ), run time (day: $F_{1,27}=71.02$; $\mathrm{p}<0.001$; Fig. 3B) and, consequently, their session duration (day: $\mathrm{F}_{1,27}=10.87 ; \mathrm{p}=0.003$ ).

\subsubsection{Session effects}

During the obstacle phase, all animals decreased their leave time (session: $F_{2,45}=4.61 ; \mathrm{p}=0.010$ ), run time (session: $\mathrm{F}_{2,45}=8.62$; $\mathrm{p}<0.001$ ) and session duration (session: $\mathrm{F}_{2,45}=5.51 ; \mathrm{p}=0.007$ ) over the three sessions.
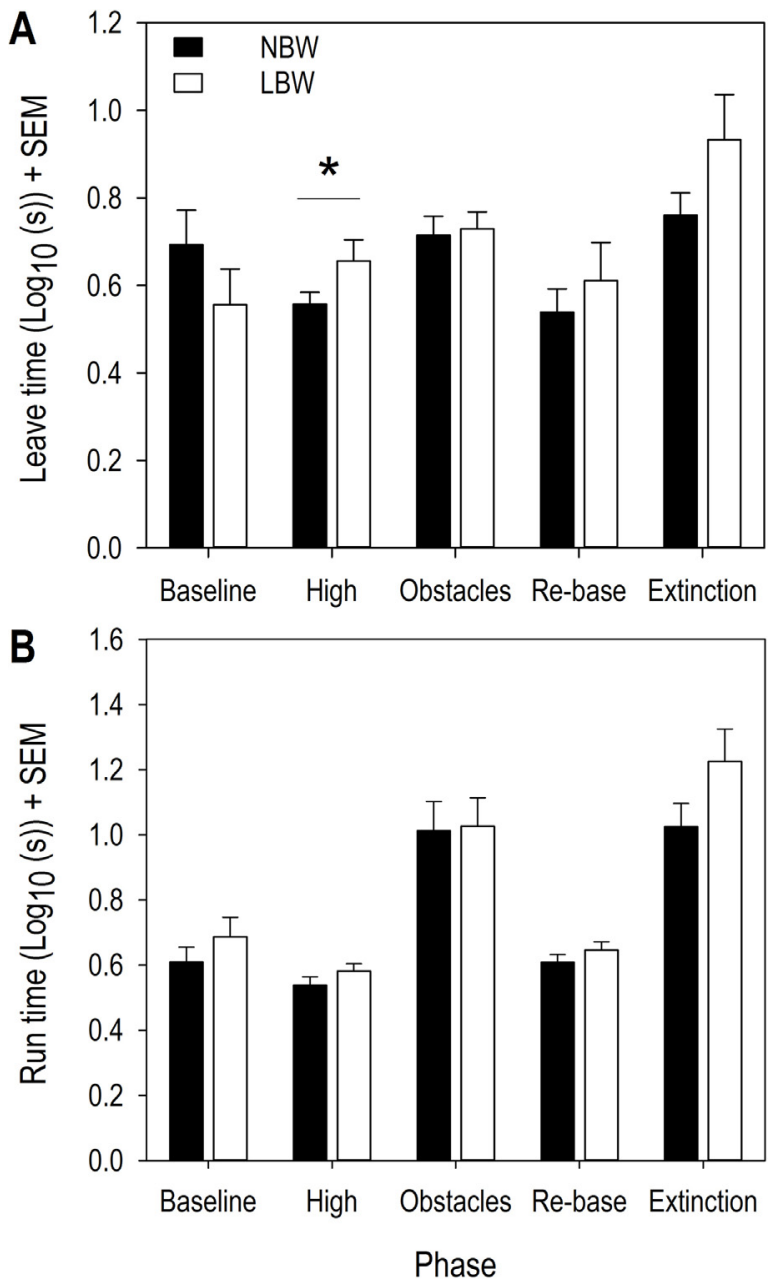

Fig. 3. Overview of the results in the runway task for all five phases (in order of testing: baseline, high reward, obstacles, re- baseline and extinction) for the low and normal birth weight pigs. Only the third day of the baseline phase is depicted in the graph. (A) Leave time in seconds; (B) Run time in seconds.

During the extinction phase, all pigs increased their leave time (session: $F_{1,27}=6.10 ; p=0.020$ ), run time (session: $F_{1,27}=13.65$; 


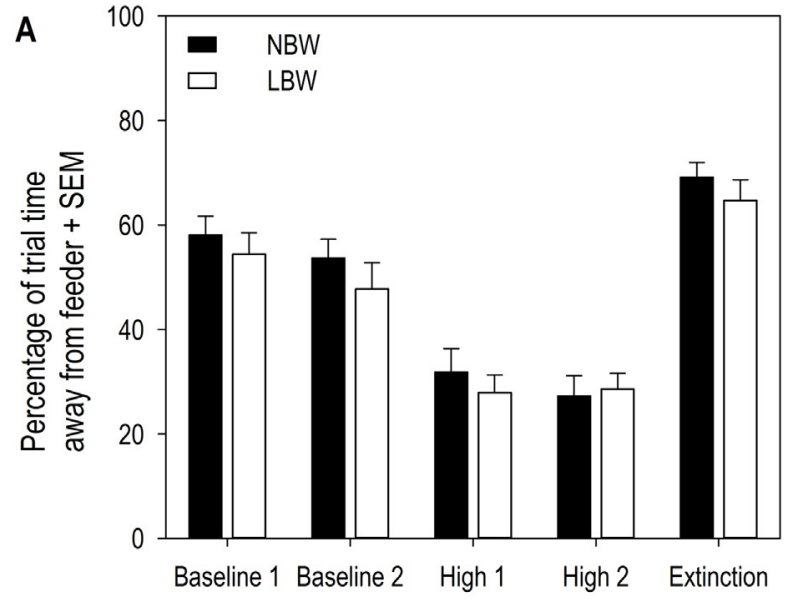

B

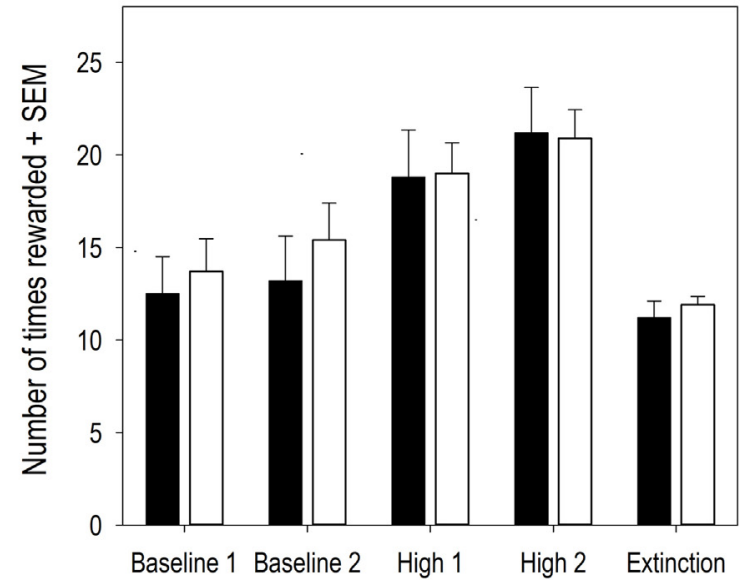

C

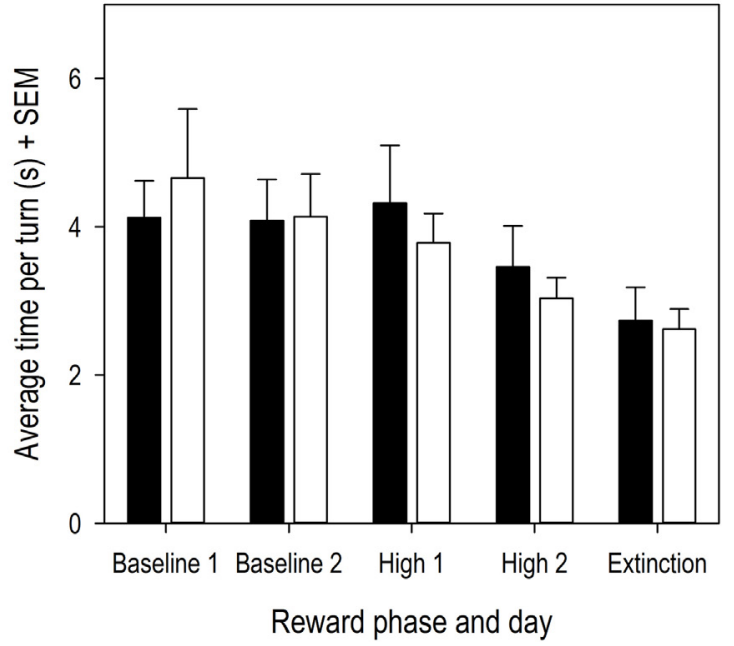

Fig. 4. Overview of the results in the nose wheel task for all phases (in order of testing: baseline, high reward and extinction) for the low and normal birth weight pigs. (A) The percentage of time away from the feeder; (B) The number of times rewarded, and (C) The average time per turn.

$\mathrm{p}=0.001$ ) and session duration (session: $\mathrm{F}_{1,27}=13.38 ; \mathrm{p}=0.001$ ) over the two sessions.

\subsection{The nose wheel task}

In the nose wheel task, no birth weight effects or interaction effects were found on any measure (Fig. 4).

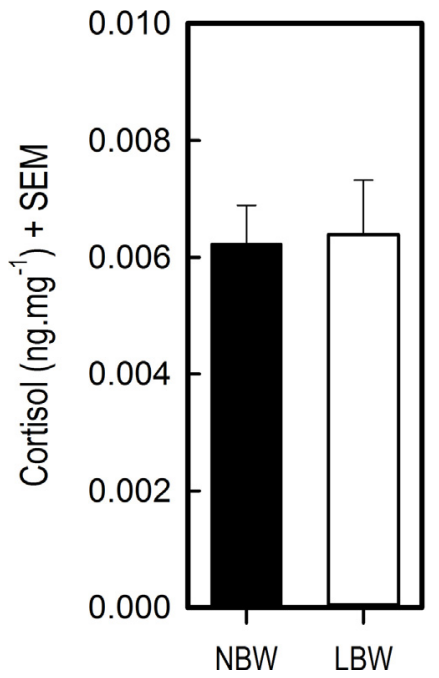

Fig. 5. Hair cortisol concentrations in $\mathrm{ng} \mathrm{mg}^{-1}$ in flank hair of normal and low birth weight pigs at 12 weeks of age.

\subsubsection{Phase effects}

The percentage of time spent away from the feeder was lower on the second baseline day for all animals compared to the first baseline day (day: $F_{1,27}=8.30 ; p=0.008$ ). The number of times rewarded and the average time per turn did not differ between baseline days (Fig. 4).

After the transition to a high reward, all animals showed a decrease in the percentage of time spent away from the feeder (day: $F_{1,27}=48.03 ; p<0.001$ ) and reached a higher number of times rewarded (day: $F_{1,27}=39.10 ; p<0.001$ ). Average time per turn did not change in this transition.

Between the first and second high reward day, percentage of time spent away from the feeder did not change. However, the number of times rewarded increased (day: $F_{1,27}=12.12 ; p=0.002$ ) and the average time per turn decreased for all animals (day: $\left.\mathrm{F}_{1,27}=19.57 ; \mathrm{p}<0.001\right)$.

After the transition from a high reward to no reward (extinction), all pigs spent a higher percentage of time away from the feeder (day: $F_{1,27}=227.27 ; p<0.001$ ). Furthermore, they reached a lower number of times rewarded, i.e. completed less required wheel turns (day: $F_{1,27}=69.72 ; p<0.001$ ) and needed less time per turn (day: $F_{1,27}=6.56 ; p=0.020$ ).

There was no birth weight effect on the number of pigs that reached the total trial duration in any phase.

\subsection{Hair samples}

No birth weight effect was found on flank hair cortisol concentration $\left(F_{1,8}=0.12 \mathrm{p}=0.740\right.$; Fig. 5).

\section{Discussion}

The present study aimed to compare the motivation to obtain food rewards between low birth weight (LBW) and normal birth weight (NBW) pigs. To this end, we used varying numbers of rewards in two separate tests; the runway task and the nose wheel task (an operant conditioning task using a progressive ratio schedule). In the runway, we found that NBW pigs had a shorter leave time in the high reward phase, but no other birth weight effects were found in either test. Both the results of the runway and the nose wheel task showed a phase effect between the baseline and high reward (increased motivation) and high reward and extinction phase (decreased motivation). Both tasks are thus sensitive to 
experimental manipulations which influence motivation for food rewards in pigs (e.g. size of reward).

\subsection{Suitability of the runway and the nose wheel task to study motivation}

We hypothesized that all pigs would show an increased motivation for a higher number of food rewards and a decrease in motivation during the extinction phase. The phase effects in both the runway and the nose wheel task confirm these hypotheses. This is in accordance with the underlying theory that animals have a higher motivation for resources that are more desired (Kirkden and Pajor, 2006), i.e. in the present study a larger food reward. This indicates that both the runway and the nose wheel task are appropriate tasks to detect motivational differences for food (rewards) in pigs.

We found differences in motivation within phases; both the run time and the session duration decreased over days during the baseline phase of the runway, indicating either an increased motivation or that the pigs learned to perform the task more efficiently. In line with these results, in the nose wheel task, the percentage of time away from the feeder decreased over the two baseline days. Moreover, in the high reward phase, the number of times rewarded increased and the average time per turn decreased over the two days. For the high reward phase, this can be explained by a learning effect, as the pigs might remember the high reward from the previous day. They are then likely to adjust their behavior, showing an increased motivation the next day.

It is possible that, despite sufficient training, pigs were still learning during the baseline phase. Alternatively, it may indicate that food motivation increased across successive days. Pigs were growing during the entire research period (Fig. 2B), which affects their feed intake and thus might influence food motivation. Therefore, another baseline phase after the high reward phase in the nose wheel task would improve our study design, since the high reward effect was now confounded with day effect and body weight. It is important to note that for both tasks the day effects were smaller than the phase effects. In addition, we found no difference in leave or run time in the runway task between the baseline and re-baseline.

In the obstacle phase of the runway, the leave time, run time and (consequently) session duration decreased over the three successive sessions. Since behaviors of the animals were not recorded during these trials, the cause of the decreased leave and run times over sessions remains speculative. Possible explanations are that the pigs became more efficient at passing the objects placed in the runway, that they showed more exploratory behavior in the first session or that their fear or restraint towards the unfamiliar objects decreased. Adding unfamiliar obstacles in a runway task may thus be a useful addition in studies assessing fearfulness or anxiety, and the speed of its decline over sessions. It would then be useful to record behaviors to determine whether the stimulus is actually perceived as fear-inducing.

All pigs showed reduced effort in the extinction phase in both tasks, with an increased leave and run time in the runway task, and a higher percentage of time away from the feeder and lower number of times rewarded in the nose wheel task. This result can be interpreted as pigs being quick to learn that effort is no longer rewarded. In addition, it demonstrates the pigs were motivated to perform the tasks for the rewards rather than performing them simply to run or turn the nose wheel.

\subsection{Birth weight effects on motivation}

Previous studies have found that LBW piglets have a faster learning rate than NBW piglets and it was suggested that this could be due to a higher motivation for food (Murphy et al., 2013; Antonides et al., 2015). Our current results do not support this hypothesis. In the runway task during the high reward phase, the leave time was shorter in the NBW pigs than in the LBW pigs. If the motivation of NBW pigs was higher than that of LBW pigs in this phase, it would be expected that they would not only leave the start box faster, but also that they would run faster. However, run time was not affected in this phase. Moreover, this was the only significant birth weight effect found on any measure in both tasks. This result alone is not a strong indicator of a motivational difference for food between LBW and NBW pigs.

Previous studies in humans and rats have found that the type of nutrients is important for the altered food preferences in SGA individuals and LBW rats, where especially carbohydrate and fatty diets are preferred (Lussana et al., 2008; Barbieri et al., 2009). Growing pigs have a high need of proteins in their diet (Campbell et al., 1984). In the current study, chocolate M\&M's ${ }^{\circledR}$ were used as a food reward. M\&M's ${ }^{\circledR}$ are relatively high in carbohydrates, but low in fat and proteins. It would be interesting for future studies to explore the effects of other food rewards with higher fat and protein contents on motivation in LBW pigs.

\subsection{Motivation tasks: critical notes}

Although the present study has indicated that the runway and the nose wheel task can be used to measure motivation, other factors possibly influencing the results must be taken into account. The body weights of the LBW pigs remained lower than the body weights of NBW pigs during the entire study, corroborating previous results (Gondret et al., 2005; Antonides et al., 2015). Any physiological difference between the LBW and NBW pigs could influence their ability to perform the tasks. However, Baxter et al. (2008) found that LBW piglets were equally vigorous as their NBW counterparts. In addition, using these two different motivation tasks makes it less likely that body size and strength influenced the results.

As an adaptive reaction in the uterus, the brains and heart of growth restricted piglets are sometimes spared and thus receive relatively more nutrients than other organs, resulting in asymmetrical growth (Amdi et al., 2013). It is important to note that the piglets used in this study did not show bodily asymmetries typical for IUGR, as IUGR piglets have reportedly more welfare complications and abnormalities than symmetrical LBW pigs (Rutherford et al., 2013). It is therefore unlikely that IUGR has affected the ability of LBW pigs to perform the tasks.

Regardless of the possible physical differences, pigs showed different rotation techniques in the task. Some pigs could rotate the disk for multiple turns with one push of their nose while other pigs needed more, less efficient pushes; one pig even used its teeth to rotate the disk. This could create a difference in the extent to which the pigs experienced the workload. Ferguson et al. (2009) also described individual differences in (mini)pigs that were subjected to an operant task, operating a lever. Because of the variation in applied techniques, it is difficult to design a task without individual variation. Even though different rotation techniques were applied in the nose wheel task, this test was still sensitive enough to measure a difference in motivation between the different phases.

The average time per turn decreased in the extinction phase compared to the high reward phase. This can be explained by the fact that the time at the feeder included the time the pigs needed to consume the food rewards. Since no rewards were given during the extinction phase, compared to eight rewards in the high reward phase, it is logical that the calculated average time per turn decreased. It would have been more accurate to subtract the time needed for consumption from this measure.

A possible drawback of food motivation tasks is that the tested animals can approach satiation during a session (Kirkden and Pajor, 
2006). This is especially of importance when a higher amount of food (rewards) is provided. However, during the nose wheel task, all pigs reached the maximum time of 900 s during the high reward phase, making it unlikely that they became satiated.

\subsection{Hair cortisol concentration}

At 12 weeks of age, flank hair cortisol did not differ between LBW and NBW pigs. Antonides et al. (2015) found significantly lower cortisol levels in flank hair of LBW pigs compared to NBW pigs, using the same method as the current study. This implies that the LBW piglets in that study experienced less stress compared to their NBW siblings. When comparing housing conditions, the pigs in that study were housed in much smaller pens than in the current study. As suggested in the discussion of that paper, the difference in cortisol between NBW and LBW animals may have been due to the relatively less space available per pig for the (larger) NBW animals.

In the current study, all pigs had ample space available. This may explain why we did not find differences in cortisol levels between birth weight groups. It is therefore advised to house pigs (and other animals used in research) in large pens or cages, in order to avoid housing conditions to influence test results. This especially accounts for experiments in which body size differs between experimental groups.

\section{Conclusion}

We found that NBW pigs had a shorter leave time in the high reward phase in the runway, but no other birth weight effects were found in either test. Therefore, we conclude that there is no motivational difference between LBW and NBW pigs for food rewards. However, both the results of the runway and the nose wheel task showed a phase effect between the baseline and high reward phase (increased motivation) and high reward and extinction phase (decreased motivation). This is in accordance with the underlying theory that animals have a higher motivation for resources that are more desired. Our results show that both the runway and the nose wheel task are sensitive enough to measure differences in motivation, thus supporting the notion that these tasks are suitable motivation tasks for pigs.

\section{Acknowledgements}

The authors would like to thank Jan van Mourik, Jan-Adriaan den Hertog, Dirk van der Heide and Serana van Laarhoven for their dedicated help taking care of the pigs. We thank Lars Ravesloot for carrying out the cortisol measurements and Elly Zeinstra and Christien Oei for their guidance and assistance in the lab work. Lastly, we are thankful for the feedback and comments of Liesbeth Bolhuis.

\section{References}

Amdi, C., Krogh, U., Flummer, C., Oksbjerg, N., Hansen, C.F., Theil, P.K., 2013. Intrauterine growth restricted piglets defined by their head shape ingest insufficient amounts of colostrum. J. Anim. Sci. 91, 5605-5613.

Antonides, A., Schoonderwoerd, A.C., Nordquist, R.E., van der Staay, F.J., 2015. Very low birth weight piglets show improved cognitive performance in the spatial cognitive holeboard task. Front. Behav. Neurosci. 9, 43.

Barbieri, M.A., Portella, A.K., Silveira, P.P., Bettiol, H., Agranonik, M., Silva, A.A., Goldani, M.Z., 2009. Severe intrauterine growth restriction is associated with higher spontaneous carbohydrate intake in young women. Pediatr. Res. 65, 215-220.

Baxter, E.M., Jarvis, S., D'Eath, R.B., Ross, D.W., Robson, S.K., Farish, M., Nevison, I.M., Lawrence, A.B., Edwards, S.A., 2008. Investigating the behavioural and physiological indicators of neonatal survival in pigs. Theriogenology 69, $773-783$.

Campbell, R.G., Taverner, M.R., Curic, D.M., 1984. Effect of feeding level and dietary-protein content on the growth, body-composition and rate of protein deposition in pigs growing from 45-kg to 90-kg. Anim. Prod. 38, 233-240.

da Silva, C.S., van den Borne, J.J., Gerrits, W.J., Kemp, B., Bolhuis, J.E., 2012. Effects of dietary fibers with different physicochemical properties on feeding motivation in adult female pigs. Physiol. Behav. 107, 218-230.

Douglas, S.L., Edwards, S.A., Sutcliffe, E., Knap, P.W., Kyriazakis, I., 2013. Identification of risk factors associated with poor lifetime growth performance in pigs. J. Anim. Sci. 91, 4123-4132.

Ferguson, S.A., Gopee, N.V., Paule, M.G., Howard, P.C., 2009. Female mini-pig performance of temporal response differentiation, incremental repeated acquisition, and progressive ratio operant tasks. Behav. Process. 80, 28-34.

Gieling, E.T., Nordquist, R.E., van der Staay, F.J., 2011. Assessing learning and memory in pigs. Anim. Cogn. 14, 151-173.

Gluckman, P.D., Hanson, M.A., 2004. Living with the past: evolution, development, and patterns of disease. Science 305, 1733-1736.

Gondret, F., Lefaucheur, L., Louveau, L., Lebret, B., Pichodo, X., Le Cozler, Y., 2005. Influence of piglet birth weight on postnatal growth performance: tissue lipogenic capacity and muscle histological traits at market weight. Livest. Prod. Sci. 93, 137-146.

Hazeleger, W., Ramaekers, P., Smits, C., Kemp, B., 2007. Effect of progenos on placental and fetal development in pigs. Reprod. Domestic Anim. 42 (135-135).

Herpin, P., Damon, M., Le Divich, J., 2002. Development of thermoregulation and neonatal survival in pigs. Livest. Prod. Sci. 78, 25-45.

Kirkden, R.D., Pajor, E.A., 2006. Using preference, motivation and aversion tests to ask scientific questions about animals' feelings. Appl. Anim. Behav. Sci. 100, 29-47.

Lussana, F., Painter, R.C., Ocke, M.C., Buller, H.R., Bossuyt, P.M., Roseboom, T.J., 2008 Prenatal exposure to the Dutch famine is associated with a preference for fatty foods and a more atherogenic lipid profile. Am. J. Clin. Nutr. 88, 1648-1652.

Martin, P., Bateson, P., 1993. Measuring Behaviour. Cambridge University Press, Cambridge.

Murphy, E., Kraak, L., Nordquist, R.E., van der Staay, F.J., 2013. Successive and conditional discrimination learning in pigs. Anim. Cogn. 16, 883-893.

Quiniou, N., Dagorn, J., Gaudre, D., 2002. Variation of piglets birth weight and consequences on subsequent performance. Livest. Prod. Sci. 78, 63-70.

Rutherford, K.M.D., Donald, R.D., Arnott, G., Rooke, J.A., Dixon, L., Mehers, J.J.M., Turnball, J., Lawrence, A.B., 2012. Farm animal welfare: assessing risks attributable to the prenatal environment. Anim. Welf. 21, 419-429.

Rutherford, K.M.D., Baxter, E.M., D’Eath, R.B., Turner, S.P., Arnott, G., Roehe, R., Ask, B., Sandøe, P., Moustsen, V.A., Thorup, F., Edwards, S.A., Berg, P., Lawrence, A.B., 2013. The welfare implications of large litter size in the domestic pig I: biological factors. Anim. Welf. 22, 199-218.

Scheel, D.E., Graves, H.B., Sherritt, G.W., 1977. Nursing order, social-dominance and growth in swine. J. Anim. Sci. 45, 219-229.

Vickers, M.H., Breier, B.H., Cutfield, W.S., Hofman, P.L., Gluckman, P.D., 2000. Fetal origins of hyperphagia obesity, and hypertension and postnatal amplification by hypercaloric nutrition. Am. J. Physiol. 279, E83-E87. 